\title{
Investigating Image Enhancement in Pseudo-Foreign Fiber Detection
}

\author{
Xin Wang ${ }^{1,3}$, Daoliang $\mathrm{Li}^{1, *}$, and Wenzhu Yang ${ }^{2}$ \\ ${ }^{1}$ College of Information and Electrical Engineering, China Agricultural University, \\ Beijing 100083, PRC \\ ${ }^{2}$ College of Mathematics and Computer, Hebei University, Baoding 071002, PRC \\ ${ }^{3}$ Computer Network Center, China Agricultural University, Beijing 100083, PRC \\ dliangl@cau.edu.cn
}

\begin{abstract}
The detection of pseudo-foreign fibers in cotton based on AVI(Automatic Visual Inspection) is crucial to improve the accuracy of statistics and classification of foreign fibers. To meet the requirement of textile factories, a new platform is introduced in which cotton bulks are floating with relative high speed of six meters per second, and the throughput of detected lint could be above $20 \mathrm{~kg}$ per hour. However, images captured by the new platform are blurred and not clear enough for post processes such as segmentation, feature extraction, target identification and statistics. Because thickness of the moving cotton bulks are not uniform, a part of or the whole object of pseudoforeign fibers are blocked. Thus image enhancement algorithms should be investigated and implemented. In this paper the characteristics of the images acquired by the new platform are analyzed, and several image enhance algorithms are studied and compared on effectiveness and efficiency, which include Histogram Equalization, Wavelet Based Normalization, Homomorphic Filtering, Single Scale Retinex(SSR), Multiscale Retinex(MSR) and Variational Retinex. Result indicated that the Variational Retinex has a better performance and should be implemented in on-line pseudo-foreign fibers detection.
\end{abstract}

Keywords: Image enhancement, Histogram Equalization, Wavelet Based Normalization, Homomorphic Filtering, Single Scale Retinex, Multiscale Retinex, Variational Retinex.

\section{Introduction}

The foreign fibers in cotton refer to those non-cotton fibers and dyed fibers, such as hairs, binding ropes, plastic films, candy wrappers, polypropylene twines, etc., which are accidentally mixed into cotton during picking, storing, drying, transporting, purchasing and processing [1]. Similarly, the pseudo-foreign fibers in cotton are some trashes such as dried cotton leaves, cotton rod, cottonseed crumbs, straws, grass stems etc. which have the same color, size and shape as the foreign fibers. Because the quantity of pseudo-foreign fibers in cotton is much larger than that of the foreign

${ }^{*}$ Corresponding author.

D. Li and Y. Chen (Eds.): CCTA 2011, Part III, IFIP AICT 370, pp. 399-409, 2012.

(C) IFIP International Federation for Information Processing 2012 
fibers, in order to improve the accuracy of foreign fibers' detection and classification with AVI (Automatic Visual Inspection), pseudo-foreign fibers should be distinguished from the real foreign fibers clearly and efficiently.

Image enhancement is an important preprocessing stage in machine vision. The aim of image enhancement is to remove the noise while retaining significant features of the image [2]. In the past few decades, many image enhancement methods such as histogram manipulation techniques, wavelet based image enhancement and PDE(Partial Differential Equations) based image enhancement have been active and open to researches. And there is no single image enhancement method applicable to all kind of images [2]. Vicent Caselles et al. invented a novel approach for shape preserving contrast enhancement by means of a local histogram equalization algorithm which preserves the level-sets of the image [3]. Wang, X.; Liu, S.; Zhou, X. proposes a new algorithm called wavelet domain Inclusive-OR denoising algorithm (WDIDA), which distinguishes the wavelet coefficients belonging to image or noise by considering their phases and modulus maxima simultaneously and in order to enhance the edges of the image but not magnify noise, a contrast nonlinear enhancing algorithm is presented according to human visual properties [4]. Farrahi Moghaddam, R.; Cheriet, M. propose a novel PDE-based method for the restoration of the degradations in single-sided document images [5].

Previous image enhancement methods work well in the foreign fiber detection platform of low throughput where the cotton layers are thinner and the image of foreign fibers are clearer and more prominent. Yang, W.Z., et al. proposed a piecewise transform model that splits the distribution range of image pixels into two or more pieces, and performs transformation to each piece respectively to enhance the region of interesting [6]. But, in order to improve the detection efficiency, a new platform is constructed in which the speed of cotton flow is above 6 meters per second, and throughput of cotton is more than $20 \mathrm{~kg}$ per hour, so the images taken by this new platform will have a background like clouds in the sky and with pseudoforeign fibers, uneven color spots and shadows mixed into them. Thus the tasks of identification are more complicated, and the probability of misclassification will be greater if we use the previous image enhancement algorithm.

The images acquired from the AVI system which contain pseudo-foreign fibers are usually not very clear because the clouds on of cotton layers. So the pseudo-foreign fiber objects are imaged under uneven lighting conditions and should be enhanced before other processes such as segmentation etc.. Many methods are developed for the enhancement of images which have cloud-like background, dim and non-uniform illumination, some of them are very successful in foreign matters detection in wool or cotton, power line detection in urban area, and face recognition under uneven lighting conditions. For example, LU Xiang-Ju, et al. posed a novel pseudo-color approach which can effectively mark all different particulars while it has the ability to divide foreign pieces with indiscernible brightness in fiber masses [7]. Jing Sun, et al. put forward a new method to detect and remove shadow from solo images of natural scenes [8]. Tang liang, et al. presented a fuzzy retinex to enhance the poor visibility of features in shadowed regions created by buildings in urban aerial images which prevents recognition of objects[9]. Although the above techniques present good results, several disadvantages of the reviewed methods still exist. For example, the contradiction between algorithm speed and precision degree exist almost in every method. 
In order to find a better algorithm (relative) to use for the enhancement of images acquired by the new platform in pseudo-foreign fiber detection, several image enhancement methods are studied on principle and effectiveness, and experiment result are showed and analyzed.

In summary, the novelties of this paper are the following:

- A new pseudo-foreign fiber detection platform is introduced.

- Several illumination improve algorithms are investigated and applied to the images produced by the new platform.

- Experiment results are compared and analyzed, and best algorithm is chosen for further studies.

The rest of the paper is organized as follows. Section 2 briefly introduce the new platform, and images samples collected by it. In Section 3, the proposed method is described in details. Section 4 describes the result and presents experimental evidence, while concluding remarks are drawn in Section 5.

\section{Image Acquisition and Samples}

\subsection{Image Acquisition}

The images used in trial are taken from the foreign fiber detection platform constructed by the Agricultural Intelligence Research Laboratory of China Agricultural University and China Cotton Machinery \& Equipment Co., Ltd. The schematic set-up of the computer vision system is presented in Fig.1.

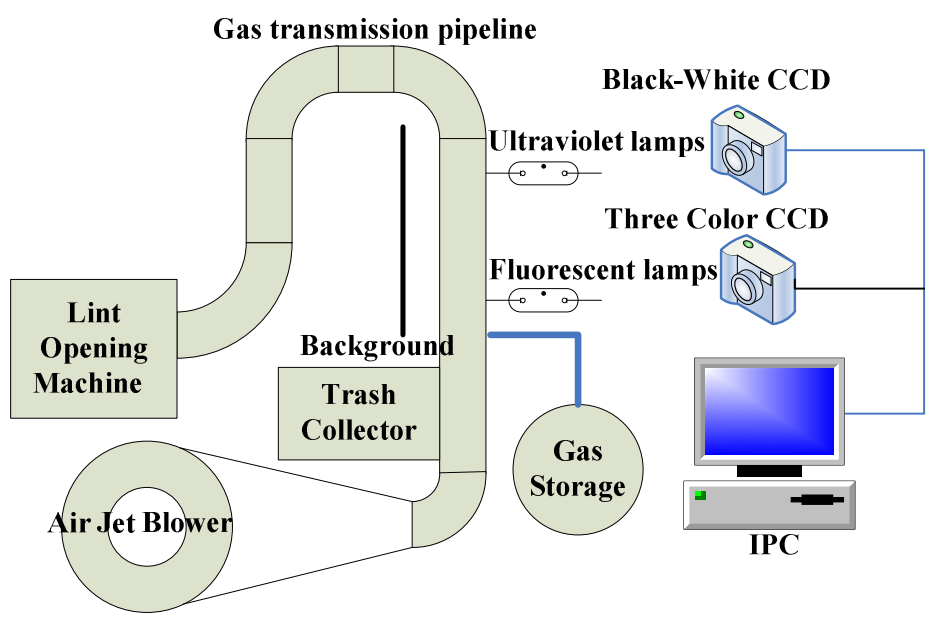

Fig. 1. Schematic set-up of the platform

The cotton processed by the Lint Opening Machine is passing through the Gas transmission pipeline then the computer triggers the flasher when an image is being grabbed by the Black-White CCD lighting by Ultraviolet lamps and the Three Color 
CCD lighting by Fluorescent lamps respectively. The image then will be processed by the algorithms installed in the computer, and both real foreign fibers and pseudoforeign fibers would be detected. The algorithms should discern pseudo-foreign fibers from real foreign fibers, and the presence of real foreign fibers will trigger the subsequent cleaning processes.

Cotton, foreign fiber, and pseudo-foreign fiber samples are provided by the China Cotton Machinery \& Equipment Co., Ltd. which are picked out by human workers, sorted and classified by engineers.

\subsection{Image Samples}

A typical image that contains pseudo-foreign fibers is presented in figure2 as an example. The size of the image is $4096 * 60$. Because the width of the image, the presentation in Fig. 2 is not very clear and we cannot discern the pseudo-foreign fiber in it, so a fraction of the image is cut out and presented as Fig.3. In this image a typical pseudo-foreign fiber maybe a grass leave is buried into the cotton layers and compared to the whole background tiny and dim.

Fig. 2. Image contains pseudo-foreign fibers

Fig. 3. A fraction of the above image

\section{Image Enhancement Methods}

By analyzing the images produced by the new platform, several features of them can be concluded:

- The lighting of the image is not even because the bulks of cotton have different thickness and they absorb and reflect light differently;

- The amount of cotton is not even in every image which make a complex background;

- Most of the pseudo-foreign fibers are buried into the cotton, so the image can only capture a part of them, and the lighting of them is not even too;

- Most of the objectives are small compared to the whole background, and have a low contrast.

In order to detect foreign matters exist in cotton by AVI, and discriminate pseudoforeign fibers from real foreign fibers, image enhance method should be implemented before other processes such as segmentation and pattern recognition. Thus several algorithms used in face recognition under uneven lighting conditions and thin line detection in cloudy weathers are studied and compared, which include Histogram equalization, Wavelet based normalization, Homomorphic filtering, SSR(Single scale Retinex), MSR(Multi-scale Retinex) and Variational Retinex. 


\subsection{Histogram Equalization}

Histograms are the basis for numerous spatial domain processing techniques. [10] Histogram equalization is a method that can usually increase the global contrast of many images, especially when the usable data of the image is represented by close contrast values. Through this adjustment, the intensities can be better distributed on the histogram. This allows for areas of lower local contrast to gain a higher contrast. $\mathrm{HE}$ is simple and effective in enhancing the low contrast image only if (a) it contains single object or (b) no apparent contrast change between object and background [11].

Suppose the gray probability density function) of image $f(x, y)$ is $p_{f}(f)$, if the gray scale on point $(\mathrm{x}, \mathrm{y})$ is $\mathrm{f}$ in original image $f(x, y)$, then we do some kind of transform to it as $g(x, y), g(x, y)=T[f(x, y)]=\int_{0}^{f(x, y)} p_{f}(u) d u$. So $\mathrm{g}$ is a probability distribution function and have attributes as follows:

- The transform from $g$ to $f$ is single-valued, monotonic, and increasing;

- $0 \leq g \leq 1$;

- $\mathrm{g}$ is a uniform random variable that distributed from 0 to 1 .

As for digital image the histogram of it with intensity levels in the range $[0, \mathrm{~L}-1]$ is a discrete function $h\left(r_{k}\right)=n_{k}$, where $r_{k}$ is the $k$ th intensity value and $n_{k}$ is the number of pixels in the image with intensity $r_{k}$. A normalized histogram is given by $p\left(r_{k}\right)=n_{k} / M N \quad k=0,1, \ldots, L-1$ where $p\left(r_{k}\right)$ is an estimate of the probability of intensity level $r_{k}$ in an image. So the gray scale on point(x,y) is $s_{k}=T\left[r_{k}\right]=\sum_{l=0}^{k} p\left(r_{k}\right)=s_{k-1}+p\left(r_{k}\right)$. Gray scales which have more pixels will be increased in differential with the previous scale after histogram equalization transform, and in general objective and background possess more pixels, hence the transform actually enhanced the contrast between the objective and the background. At the same time gray scales have little pixels will be decreased in differential with the previous scale after histogram transform, usually the number of pixels in the transition between border and background are small and they are incorporated either in border or in background, therefore the border will be more sharp after transform, so the contrast of image is enhanced.

Here we applied the transformation to all intensity levels, so the resulting intensities have a uniform PDF (probability density function). And all pixels in an image are ordered from the most negative to the most positive (from the one with the smallest intensity value to the one with the largest intensity value).There are other histogram equalization algorithms with different PDF and shape-preserving local histogram equalization, and we shall discuss them in other papers. 


\subsection{Wavelet Based Normalization}

A wavelet is a wave-like oscillation with amplitude that starts out at zero, increases, and then decreases back to zero. It can typically be visualized as a "brief oscillation" like one might see recorded by a seismograph or heart monitor. Generally, wavelets are purposefully crafted to have specific properties that make them useful for signal processing. The main advantage of wavelet analysis is that it can provide abilities of local analysis and refinement in both spatial and frequency domain. And as for high frequency signals it adopt a gradually meticulous steps in frequency or spatial domain, so wavelet can focus on and be used to analyze any detail of objects in a signal or image. The formula of Continuous wavelet transforms is as follows.

$$
c(s, p)=\int_{-\infty}^{\infty} f(t) \varphi(s, p) d t
$$

The results of Continuous wavelet transforms are many wavelet coefficients which are called scale factor $\mathrm{s}$ and translation function $\mathrm{p}$ respectively. If every possible scales or translation coefficients are calculated, the work will be too hard to accomplish, actually only scale factors and translation coefficients that are times of $2^{j}$ are chosen, thus the data for analysis would be decreased dramatically. And this kind of calculates are called Discrete wavelet transforms, in general Discrete wavelet transforms are also named dual-scale wavelet.

After the process of wavelet coefficients, the signal should be restored to its original form which is called wavelet restoration, wavelet synthesis or inverse wavelet transform.

The fundamental principle for wavelet transform used in image enhancement is that the coefficients matrix result from wavelet transform represent different frequency traits of image, by multiply them with different enhance factors different traits are enhanced, then through the inverse wavelet transform some part of the images can be enhanced.

Wavelet transfer has been used in many fields of image processing such as image enhancement, restoration, de-noising, compression and so on. All wavelet transforms may be considered forms of time-frequency representation for continuoustime (analog) signals and the method used in this tests equalizes the histogram of the approximation coefficients matrix and emphasizes (by scaling) the detailed wavelet coefficient in the three directions. As a final step it performs an inverse wavelet transform to recover the enhanced image.

In general wavelet can enhance the detail information in an image while give rise to a lot of noises, thus reduce the visual effect.

\subsection{Homomorphic Filtering}

Homomorphic filter is based on the theory which separate image into luminance component $L(x, y)$ and reflection component $\mathrm{r}(\mathrm{x}, \mathrm{y})$ like the Retinex. 


$$
S(x, y)=R(x, y) \otimes L(x, y)
$$

In an image luminance can be represented by a gradually changed space while reflection tend to be expressed by a rapid changed space. Thus we can relate the low frequency of log domain of an image to the luminance component and the high frequency of $\log$ domain to the reflection component. Because the luminance represent the dynamic range of pixels in an image while contrast of an image tend to be function of its reflection component, Homomorphic filter can achieve ideal control to both components of the image by decreasing the low frequency and increasing the high frequency at same time. As a result the process of homomorphic filter can both compress the dynamic range of pixels and enhance the contrast of an image. The flow chat of homomorphic filtering is presented as figure.

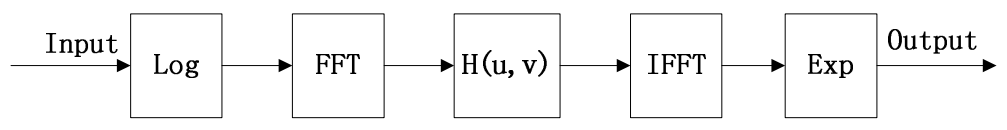

Fig. 4. Flow chart of Homomorphic filtering

The algorithm proposed first transform the image into the logarithm and then into the frequency domain. Here, the high frequency components are emphasized and the low-frequency components are reduced. As a final step the image is transformed back into the spatial domain by applying the inverse Fourier transform and taking the exponential of the result[12].

In practice ideal low pass and high pass filter used in homomorphic filtering which suitable for all kinds of image are hard to find, so the homomorphic filter build on the bases of Fourier transform sometime can't achieve good results .

\subsection{Single Scale Retinex and Multi Scale Retinex}

SSR and MSR are based on the theory of Retinex proposed by Land in the year 1963 which is an image enhancement and illumination compensation model of the lightness and color perception of human vision[4]. "Retinex" is a compound word came from Retina and Cortex. It is believed that our vision system is subjective when it comes to color perception. Our vision ensures that the perceived color of objects remains relatively constant under varying illumination conditions[13]. Because the principal of Retinex excludes the influences of variant lighting, a series of algorithm is developed based on this theory to detect objectives under uneven lighting or Remote Sensing images in cloudy day or other light lacking conditions.

The formula of Retinex is showed in equation (3).

$$
S(x, y)=R(x, y) \otimes L(x, y)
$$

The digital image $\mathrm{S}$ can be separated into two images: the reflectance $\mathrm{R}$ and the illumination $\mathrm{L}$. Then the problem is that $\mathrm{L}$ should be estimated from $\mathrm{S}$, and $\mathrm{R}$ is the 
enhanced image needed. There are many ways to calculate L from S, SSR and MSR are two of them and the detail of other algorithms would be discussed in other papers.

SSR and MSR are based on the principal of center/surround and the main idea of which is that the illumination component tends to change more smoothly than the surface reflectance, so this algorithm involves computing an average weighted by the distance from the point in question, and subtracting the logarithm of this average logarithm of the lightness [14].

$$
\begin{gathered}
\log [R(x, y)]=\log \frac{S(x, y)}{L(x, y)}=\log [S(x, y)]-\log [S(x, y) \otimes G(x, y)] \\
G(x, y)=\lambda \bullet e^{\frac{-\left(x^{2}+y^{2}\right)}{c^{2}}}
\end{gathered}
$$

$\lambda$ is a constant matrix which makes the equation (4) true.

$$
\iint G(x, y) d x d y=1
$$

The formula of MSR is showed as below:

$$
r(x, y)=\sum_{i=1}^{N} w_{i}\left\{\log [S(x, y)]-\log \left[S(x, y) \otimes G_{i}(x, y)\right]\right\}
$$

$\mathrm{N}$ represents the number of scales, normally $\mathrm{N}$ is equal to $3, W_{i}$ is the weighting coefficient. Here $W_{i}$ is $1 / 3$. The parameter $\mathrm{c}$ in equation (3) is denoted as the scale of the algorithm, and experiments indicate that the best multi scales in MSR are 15, 80 and 200 respectively.

\subsection{Variational Retinex}

Variational Retinex is shown to be able to unify previous methods, leading to a new illumination estimation algorithm. Although it performs well, the computational complexity of this model is relatively high, [15] and quite a few algorithms are proposed to speed-up the method [16]. In this research calculus specifically EulerLagrange differential equation of variations are used, and the formulas is:

$$
\begin{aligned}
& \text { Minimize: } F[l]=\int_{\Omega}\left((|\nabla l|)^{2}+\alpha(l-s)^{2}+\beta|\nabla(l-s)|^{2}\right) d x d y \\
& \text { Subject to: } l \geq s, \text { and }\langle\nabla l, \vec{n}\rangle=0 \quad \text { on } \quad \partial \Omega
\end{aligned}
$$

The illumination estimation problem can be formulated as a Quadratic Programming (QP) optimization problem. Here $\partial \Omega$ represents the image, 1 denotes the illumination component, s is the original image $\Omega$, represents the edge of the image, $\beta$ a and are non-negative coefficients. 


$$
\frac{\partial F[l]}{\partial l}=0=-\Delta l+\alpha(l-s)-\beta \Delta(l-s)
$$

To solve equation (6), the partial derivative of $\mathrm{F}$ on 1 should be zero, and there are various numerical solutions to calculate 1 from formula (7). Here the idea of the steepest descent with an auxiliary variable is applied, and the initial value of 1 can be the image s. Through iterations of a given times we can get the estimation of the illumination image, and then the reflectance image or enhanced image can be calculated just as SSR .

\section{$4 \quad$ Results and Discussion}

The effect of six algorithms and their histogram are showed as follows.

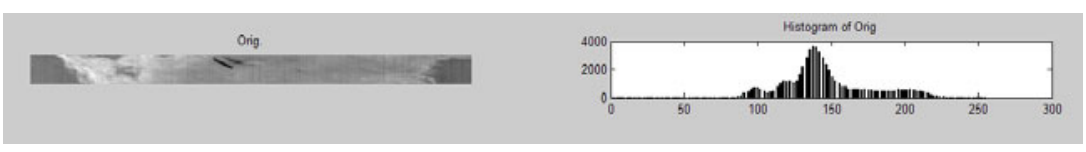

Fig. 5. The Original image and its Histogram

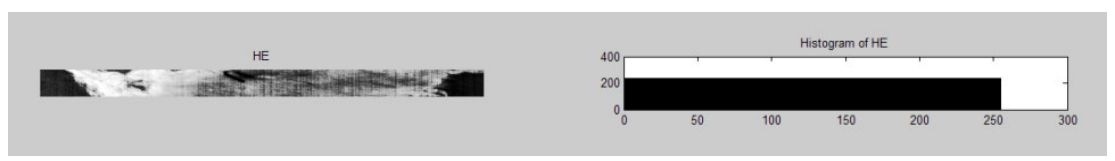

Fig. 6. Image use Histogram Equalization and its Histogram

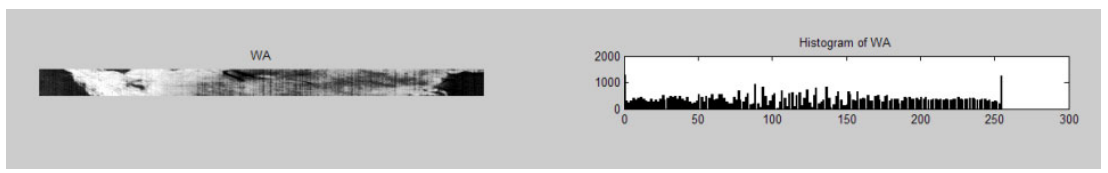

Fig. 7. Image after wavelet Normalization and its histogram

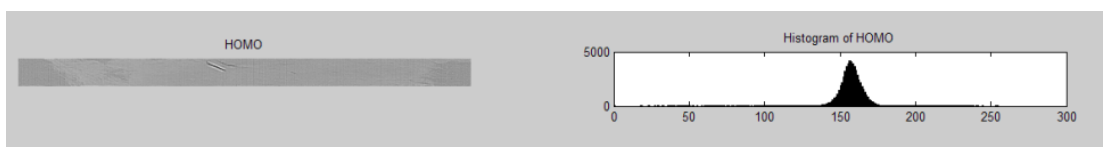

Fig. 8. Image by Homomorphic Filtering and its histogram

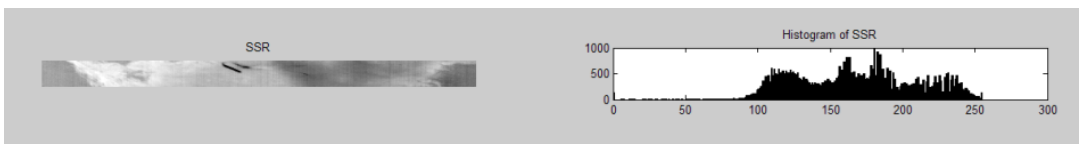

Fig. 9. Image with SSR and its histogram 


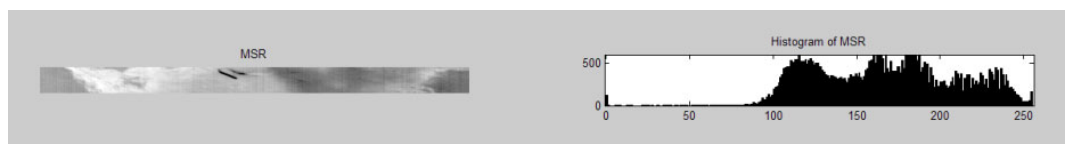

Fig. 10. Image with MSR and its histogram

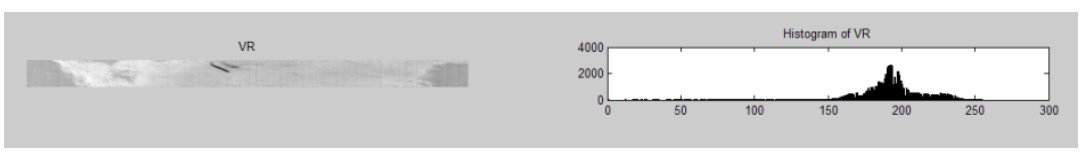

Fig. 11. Image with Variational Retinex and its histogram

By testing 40 images the average time cost of each algorithm is showed in Table1.The test images' size are $1000 * 60$ pixels. The algorithms is implement in Matlab R2010a running on a PC with Intel(R) Core(TM) 2 Quad CPU Q6600 @ $2.4 \mathrm{GHz} 2.39 \mathrm{G} \mathrm{Hz}$, memory $3.25 \mathrm{~GB}$. If the algorithms is converted to $\mathrm{C} / \mathrm{C}++$ programs the speed of execution would be significantly increased, and if a DSP chip is designed and implemented the speed could be even faster than the $\mathrm{C} / \mathrm{C}++$ programs.

Table 1. Algorithms' Average speed

\begin{tabular}{c|c}
\hline Algorithm name & Average speed $($ s $)$ \\
\hline Histogram Equalization & 0.010004 \\
Wavelet Normalization & 0.049369 \\
Homomorphic Filtering & 0.036915 \\
Single Scale Retinex & 0.634774 \\
Multi Scale Retinex & 1.929681 \\
Variational Retinex & 0.280129 \\
\hline
\end{tabular}

Experiment shows that the Histogram Equalization is much faster than other algorithms, but the enhanced image have more noises which came from the magnification of cotton shadows in the global enhance process.

The Wavelet Normalization is fast too, although the global frequency transform enhance the noises and shadows just as the Histogram Equalization.

The Homomorphic Filtering algorithm can smooth the edge between cotton bulk and the background, therefore make the foreign matters in the image more prominent. But verges of the foreign matter are blurred which are not conducive to object segmentation. And improvement of it should be investigated in the following works.

The SSR and MSR have the same enhancement effectiveness, but the speed of them are not suitable for online detection.

The Variational Retinex's comprehensive performance is better in object enhancement, shadow removal and process speed. 


\section{Conclusion}

In this paper, a new foreign fiber detection platform is introduced. Images collected by the platform are studied and analyzed. Several methods are investigated for image enhancement in pseudo-foreign fiber detection, their enhance effect and algorithm speed are compared. Results indicate that the Variational Retinex is suitable for on-line pseudoforeign fiber detection. Improvement on speed of it should be studied in further researches.

Acknowledgements. The authors thank National Natural Science Foundation of China (30971693) and Ministry of Education of People's Republic of China (NCET09-0731), for their financial support, and China Cotton Machinery \& Equipment Co., Ltd. for providing foreign fiber samples and technical support.

\section{References}

[1] Yang, W., et al.: Fast recognition of foreign fibers in cotton lint using machine vision. Mathematical and Computer Modelling 54(3-4), 877-882 (2011)

[2] Tasmaz, H., Ercelebi, E.: Image enhancement via space-adaptive lifting scheme exploiting subband dependency. Digital Signal Processing 20(6), 1645-1655 (2010)

[3] Sengur, A., Guo, Y.: Color texture image segmentation based on neutrosophic set and wavelet transformation. Computer Vision and Image Understanding 115(8), 1134-1144 (2011)

[4] Wang, X., Liu, S., Zhou, X.: New algorithm for infrared small target image enhancement based on wavelet transform and human visual properties. Journal of Systems Engineering and Electronics 17(2), 268-273 (2006)

[5] Farrahi Moghaddam, R., Cheriet, M.: RSLDI: Restoration of single-sided low-quality document images. Pattern Recognition 42(12), 3355-3364 (2009)

[6] Yang, W.Z., et al.: A new approach for image processing in foreign fiber detection 68(1), 68-77 (2009)

[7] Lu, X., Ding, M., Wang, Y.: A New Pseudo-color Transform for Fibre Masses Inspection of Industrial Images. Acta Automatica Sinica 35(3), 233-238 (2009)

[8] Sun, J., Du, Y., Tang, Y.: Shadow Detection and Removal from Solo Natural Image Based on Retinex Theory. In: Xiong, C.-H., Liu, H., Huang, Y., Xiong, Y.L. (eds.) ICIRA 2008. LNCS (LNAI), vol. 5314, pp. 660-668. Springer, Heidelberg (2008)

[9] Tang, L., et al.: Removing Shadows from Urban Aerial Images Based on Fuzzy Retinex. Acta Electronica Sinica 33(3), 500-503 (2005)

[10] Digital Image Processing Third Edition, p.142 (2010)

[11] Cheng, H.D., Shi, X.J.: A simple and effective histogram equalization approach to image enhancement. Digital Signal Processing 14(2), 158-170 (2004)

[12] An Investigation of Retinex Algorithms for Image Enhancement. Journal of Electronics (China) (05), 696-700 (2007)

[13] Almoussa, N.: Variational Retinex and Shadow Removal. The Mathematics Department, UCLA (2008)

[14] Inface: A Toolbox for Illumination Invariant Face Recognition Toolbox description (2009)

[15] An Investigation of Retinex Algorithms for Image Enhancement. Journal of Electronics(China) (05), 696-700 (2007)

[16] A fast algorithm for color image enhancement with total variation regularization. Science China (Information Sciences) (09), 1913-1916 (2010) 